05

\title{
Интерференция нейтронных волн при малоугловом рассеянии на ферромагнитных сплавах
}

\author{
(C) А.В. Ковалев \\ Петербургский институт ядерной физики им. Б.П. Константинова НИЦ „Курчатовский институт“, \\ Гатчина, Россия \\ E-mail: kovalev_av@pnpi.nrcki.ru \\ (Поступила в Редакцию 26 января 2018 г.)
}

На намагниченных пластинках сплава $\mathrm{Co}_{67} \mathrm{Fe}_{31} \mathrm{~V}_{2}$ исследовались условия обнаружения и особенности интерференции прямых и рассеянных нейтронных волн. Для противоположных направлений поляризации исходного пучка измерялись угловые распределения интенсивностей прошедших через образец нейтронов. Признаком изучаемого эффекта, который наблюдается при наличии в образцах магнитного рассеяния на неоднородностях кристаллической структуры, являются разные интегральные интенсивности пиков „без переворота спина нейтрона“. Отношение этих интенсивностей зависит от режима термической обработки образца, его толщины и величины приложенного к нему магнитного поля. Происхождение пиков „с переворотом спина нейтрона“ связано с механизмом прохождения нейтронных волн через магнитнонеколлинеарные границы. Излагаются методы измерений и обработки экспериментальных данных.

DOI: 10.21883/FTT.2018.10.46519.026

\section{1. Введение}

Примеры использования малоуглового рассеяния поляризованных нейтронов (MP или SAPNS) для определения параметров наночастиц изложены в статьях [1,2]. Для однозначного решения подобных задач необходима достаточная степень монодисперсности частиц и малая их концентрация в однородной матрице. Выполнение этих условий для ферромагнитных сплавов маловероятно. Однако метод МР позволяет, в частности, изучать процессы формирования мелкодисперсных фаз при термообработке магнитных сплавов. Сравнение экспериментальных данных, полученных на сплавах $\mathrm{Co}_{67} \mathrm{Fe}_{31} \mathrm{~V}_{2}$ и $\mathrm{Co}_{68} \mathrm{Fe}_{32}$, показало [3], что при их анализе необходимо учитывать эффект, причиной которого предлагалось считать интерференцию нейтронных волн в области расходимости прямого пучка [4].

Возможность наблюдения такого эффекта связана с тем, что разность фаз прямой и рассеянной волн равна $\pi$. Но достаточная степень коллинеарности соответствующих волновых векторов для ферромагнитных сплавов казалась невозможной. В литературных источниках результатов исследований в этом направлении не обнаружено. Исходная цель наших исследований изложена в статье [3]. Измерения выполнялись на установке „Вектор“ (реактор ВВР-М, Гатчина).

\section{2. Образцы и общая схема обработки экспериментальных данных}

Пластинки сплава $\mathrm{Co}_{67} \mathrm{Fe}_{31} \mathrm{~V}_{2}$ оказались удобными для выполнения настоящей работы. Во-первых, для него $A^{+} \gg A^{-}\left(A^{+}\right.$и $A^{-}$- амплитуды когерентного рассеяния для двух направлений спина нейтрона), что позволяет наблюдать максимальную величину интерференционного эффекта. Во-вторых, результаты измерений зависят от режима термической обработки образцов с примесью ванадия. Кроме того, малые коэрцитивные поля сплавов позволяют выполнять повторные измерения, а для получения дополнительной информации использовать разное число пластинок, вырезанных из одной мишени для магнетронного распыления. Контрольные опыты выполнялись на образцах без примеси ванадия.

Описания установки „Вектор“ с многоканальной детекторной системой приведено в статье [5] и обзоpe [6]. Средняя длина волны нейтронов $\langle\lambda\rangle \sim 0.92 \mathrm{~nm}$, $\Delta \lambda / \lambda_{\max } \sim 0.25$, а максимальная интенсивность спектра соответствует $\lambda_{\max } \sim 0.8 \mathrm{~nm}$. Измеренное после рассеяния образцом угловое распределение интенсивности „без переворота спина нейтрона“ (S $\uparrow \uparrow \mathbf{H})$ обозначим символом $J(00)$, а „с переворотом спина“ $(\mathbf{S} \uparrow \downarrow \mathbf{H})-$ символом $J(01)$. Для исходного состояния $\mathbf{S} \downarrow \downarrow \mathbf{H}$ получим, соответственно, $J(11)$ и $J(10)$. Направление спина $\mathbf{S}$ в первичном пучке определялось после измерения интенсивностей отраженных намагниченной пленкой нейтронов: при $\mathbf{S} \uparrow \uparrow \mathbf{H}$ должно быть $J(00) \gg J(11)$. После замены детекторной системы на двухкоординатный детектор [7] измерялись суммы интенсивностей $J($ up $)=J(00)+J(01)$ и $J($ down $)=J(11)+J(10)$.

Результаты измерений МР обычно приводятся в зависимости от величины вектора рассеяния $\mathbf{q}=\mathbf{k}_{0}-\mathbf{k}$, где $\mathbf{k}_{0}$ и $\mathbf{k}$ - волновые векторы падающей и рассеянных волн, $\left|\mathbf{k}_{0}\right|=|\mathbf{k}|=2 \pi / \lambda$, и угла $\alpha$ между $\mathbf{q}$ и $\mathbf{H}$. При этом из рассмотрения исключается область расходимости первичного пучка, что позволяет вместо символов „,“ и „1“ использовать символы „,+“ и ,-““.

Уникальные возможности поляризованных нейтронов связаны с интерференцией нейтронных волн на магнитном атоме, следствием чего являются разные интен- 

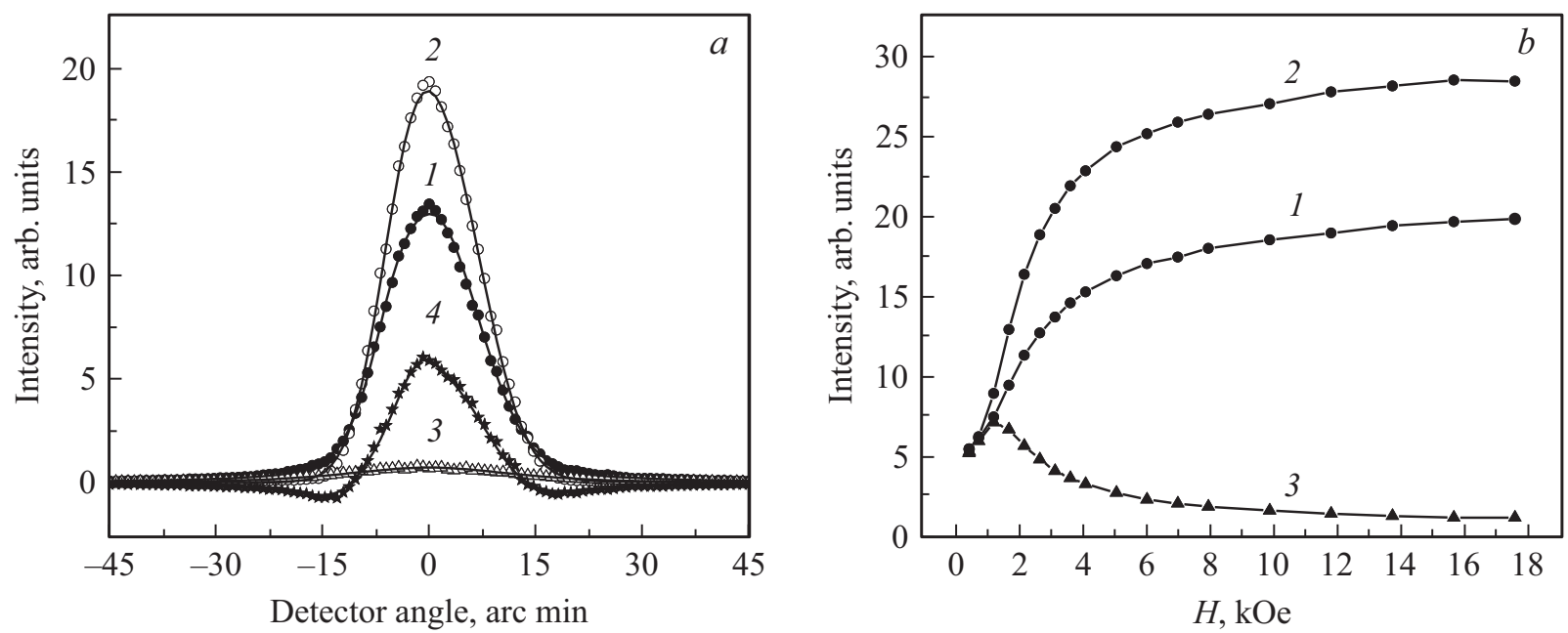

Рис. 1. а) Угловые зависимости интенсивностей $J(00)(1), J(11)(2), J(01)(3)$ и разности $\Delta J=[J(11,2 \theta)-J(00,2 \theta)](4)$ для сплава $\mathrm{Co}_{67} \mathrm{Fe}_{31} \mathrm{~V}_{2}$, отожженного при $\left.500^{\circ} \mathrm{C} . b\right)$ Соответствующие изменения интенсивностей в центральном счетчике угломерной системы при намагничивании образца.

сивности рассеяния для противоположных направлений спинов нейтронов. Для намагниченного образца

$$
\begin{gathered}
J^{++}(q, \alpha)=F_{N}^{2}+\left[F_{M}^{2}+2 F_{N} F_{M}\right] \sin ^{2} \alpha, \\
J^{--}(q, \alpha)=F_{N}^{2}+\left[F_{M}^{2}-2 F_{N} F_{M}\right] \sin ^{2} \alpha, \\
\Delta J=J^{++}(q, \alpha)-J^{--}(q, \alpha)=4 F_{N} F_{M} \sin ^{2} \alpha,
\end{gathered}
$$

где $F_{N}$ и $F_{M}$ - ядерные и магнитные части результирующих амплитуд рассеяния, пропорциональные соответствующим разностям $\Delta \eta_{j}$ амплитуд „частицы“ и матрицы [1], $\alpha$ - угол между направлениями q и $\mathbf{H}$.

В настоящей работе коллимация нейтронного пучка задавалась вертикальными щелями. Угол рассеяния $2 \theta$ (detector angle) отсчитывался вдоль горизонтальной компоненты $q_{x}=2 \pi \sin (2 \theta) / \lambda$. Для каждого интервала усреднения $\Delta \theta$ необходимо измерять суммы интенсивностей во всем интервале углов МР вдоль направления $q_{y}$. (О выполнении этого условия будет говориться далее.)

Количественной мерой интерференционного эффекта $N$ будем считать отношения интегральных интенсивностей $Q(11) / Q(00)$ или $Q($ down $) / Q($ up $)$, а величину $P_{Q}=[Q(11)-Q(10)] /[Q(11)+Q(10)]$ назовем „поляризацией“ прошедшего через образец нейтронного пучка. (Поляризацией $P$ обычно называют величины отношений $P(00)=[J(00)-J(01)] /[J(00)+J(01)]$ или $P(11)=[J(11)-J(10)] /[J(11)+J(10)]$, измеренных при $2 \theta \sim 0^{\circ}$.) При $F_{M} \neq 0$ интегральные интенсивности рассеянных нейтронов $Q^{++}>Q^{--}$, но при измерении $Q(\mathrm{ij})$ регистрируются и нейтроны, которые прошли через образец без рассеяния. Поэтому в рамках классических представлений естественно предположить, что $Q(00)=Q(11)$. Действительно, малые отличия этих интенсивностей $N \sim 1.02$ обнаружены для некоторых образцов. Максимальная же величина $N=3.1(2)$ получена на сплаве ЮНДК (ALNICO), в котором име- ется упорядоченная структура двух фаз с периодом $D=55(2)[8]$.

При вычислении интенсивностей $J(\mathrm{ij})$ учитывались систематические погрешности детекторной системы установки „Вектор“ [9]. Для сравнительного анализа наиболее удобными оказались результаты измерений на двух образцах сплава $\mathrm{Co}_{67} \mathrm{Fe}_{31} \mathrm{~V}_{2}$, которые отличались лишь разными режимами термической обработки.

\section{3. Эксперимент и обсуждение результатов}

3.1. Измерения на установке с многоканальной детекторной системой. На рис. $1, a$ показаны результаты измерений на образце № 1 толщиной $1.2 \mathrm{~mm}$, который $6 \mathrm{~h}$ отжигался при $550^{\circ} \mathrm{C}$ и $13 \mathrm{~h}$ равномерно охлаждался до $120^{\circ} \mathrm{C}$. Горизонтальное магнитное поле $H=17.6 \mathrm{kOe}$ параллельно плоскости пластинки, которая перпендикулярна направлению нейтронного пучка. Полуширина прямого пучка $W(\mathrm{Nb}) \sim 10^{\prime}$. Параметры пиков $J(00)$ и $W(11)$ задаются размерами рассеивающих „частиц“ и толщиной образца вдоль направления нейтронного пучка. Характерный вид разности $\Delta J=[J(11,2 \theta)-J(00,2 \theta)]$ - признак магнитного рассеяния в образце. Изменение знака $\Delta J$ происходит потому, что для рассеянных нейтронов $J^{--}(q)<J^{++}(q)$, но в области расходимости прямого пучка $J(11,2 \theta)>J(00,2 \theta)$. Если в образце имеется интерференции прямых и рассеянных нейтронных волн, то $\Sigma \Delta J[\Delta(2 \theta)] \neq 0$.

Большие величины полуширин $W(10) \sim W(01)=$ $=29.0^{\prime}(5)-$ результат преломления нейтронных волн на магнитно-неколлинеарных границах [10]. Вероятность переворота спина нейтрона при прохождении границы между областями с индукциями $\mathbf{B}_{1}$ и $\mathbf{B}_{2}$ равна $\sin ^{2}(\varphi / 2)$, 

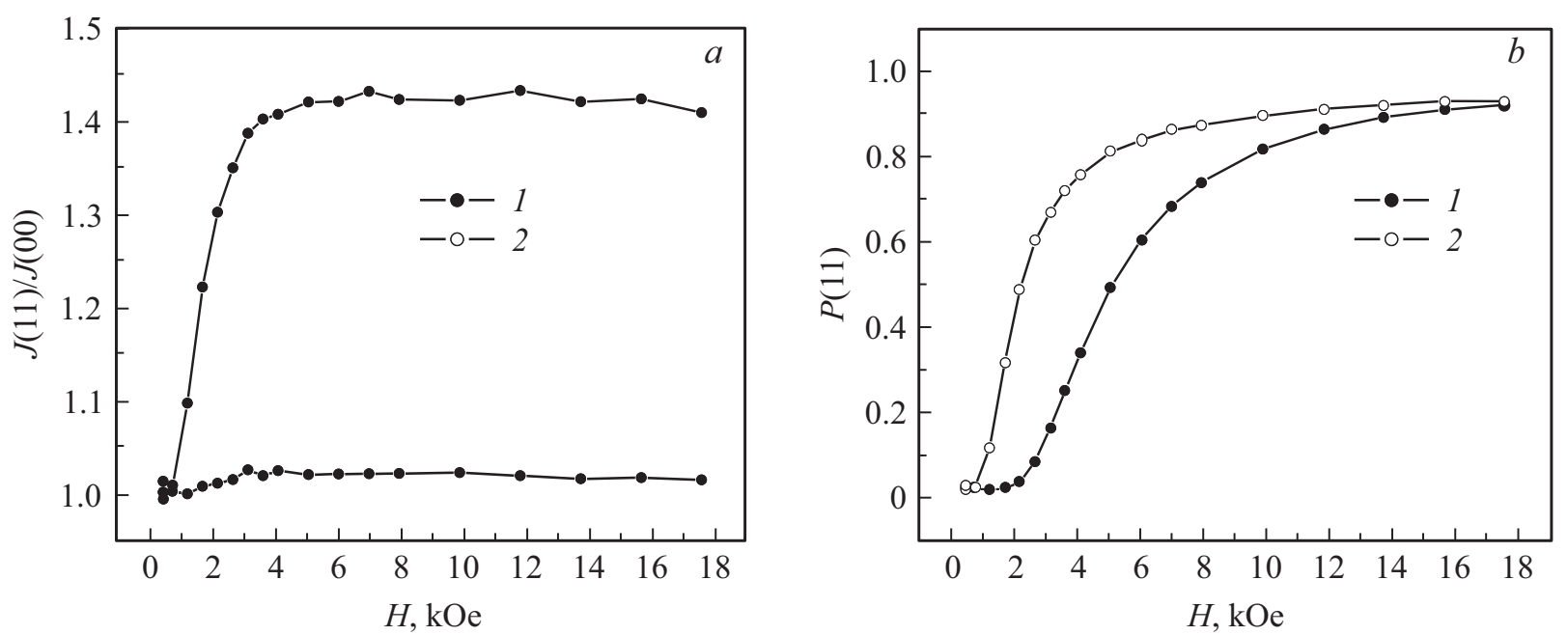

Рис. 2. Полевые зависимости параметров для исходного (1) и отожженного (2) состояний образца: $a-M(H)=J(11) / J(00)$; (b) $-P(11)=[J(11)-J(10)] /[J(11)+J(10)]$.
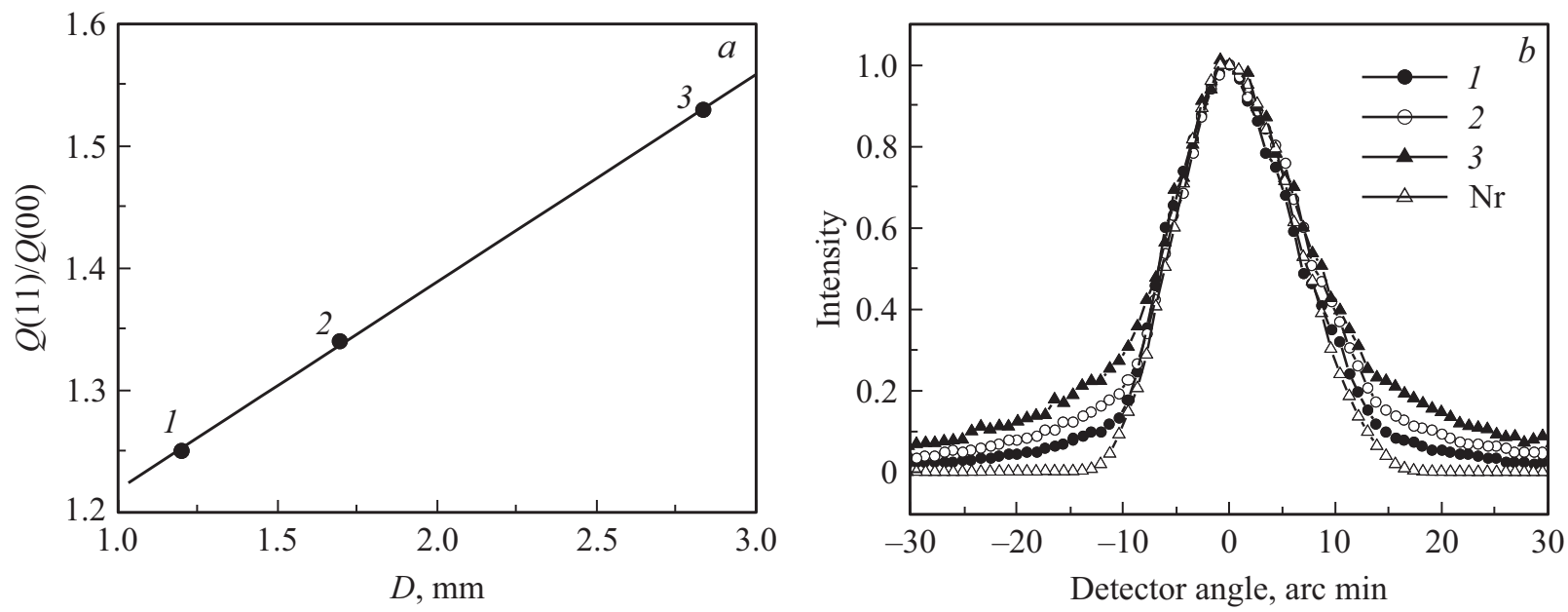

Рис. 3. $a)$ Зависимость $N(D)$, полученная при вращении магнита с образцом вокруг вертикальной оси. $b$ ) Нормированные интенсивности $J(00)$, соответствующие величинам $D$.

где $\varphi$ - угол между направлениями указанных векторов. Вид кривых, показанных на рис. $1, b$, связан с перестройкой доменной структуры при намагничивании образца, что проявляется в зависимостях $N(H)$ и $P_{Q}(H)$. При $H$ равным $0.4,3.07,4.65$ и $17.6 \mathrm{kOе}$ получилось: $N$ равно $1.00,1.22,1.25$ и 1.25 , а $P_{Q}$ равно $0.00,0.35,0.57$ и 0.83 .

Полевые зависимости параметра $M=J(11) / J(00)$ и поляризации $P(11)=[J(11)-J(10)] /[J(11)+J(10)]$ (рис. 2) можно использовать для изучения процессов намагничивания. Быстрый рост кривых $M(H)$ до $H \sim 3 \mathrm{kOe}$ связан с формированием однонаправленной магнитной текстуры, затем происходят повороты намагниченностей в отдельных доменах. Вероятность переворота спина нейтрона падает с ростом намагниченности образца, что и наблюдается в поведении $P(H)$. Для исходного состояния образца $N=0.03(1)$.

Кажется очевидным, что поляризация „рассеянного“ пучка нейтронов должна падать с ростом толщины об- разца. Однако при вращении магнита с намагниченным образцом $(H=4.65 \mathrm{kOe})$ вокруг вертикальной оси обнаружен интересный эффект. При росте толщины образца $D$ вдоль направления нейтронного пучка наблюдалась показанная на рис. $3, a$ линейная зависимость $N(D)$, но величины $P_{Q}=0.56(1)$ и $P(11)=0.77(1)$ оставались неизменными. Такой результат получится, если при вращении образца в нем сохраняется число магнитнонеколлинеарных границ вдоль направления прямого пучка. Показанные же на рис. $3, b$ изменения формы пиков $J(00)$ - признак того, что величина $N$ как-то связана с МР рассеянием на большие углы.

Для наборов одинаковых пластинок толщиной $0.6 \mathrm{~mm}$ при изменении $N$ от 1.05 до 1.22 (рис. 4, $a$ ) получилась обычная экспоненциальная зависимость (рис. $4, b)$. Коэффициент поглощения для наших образцов $\mu=1.154(5) \mathrm{mm}^{-1}$, что приводит к ослаблению первичного пучка одной пластинкой в два раза. Для пакета 

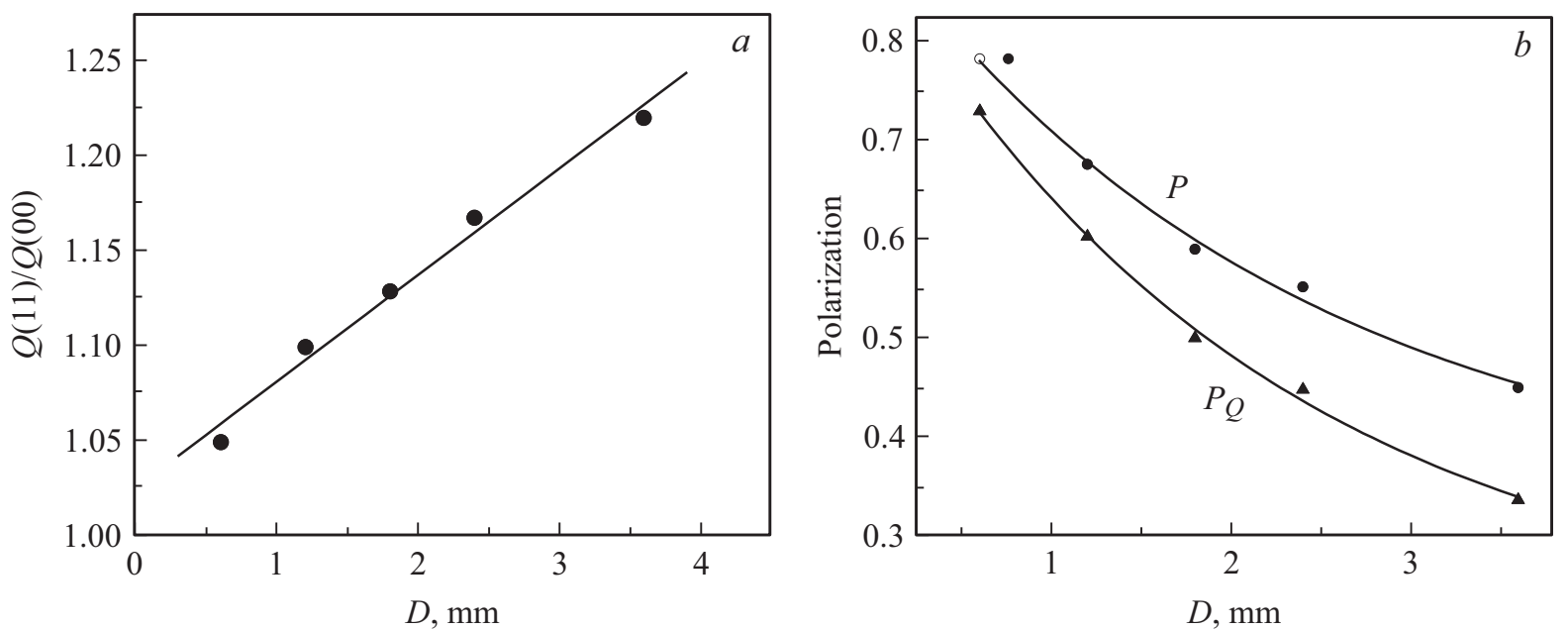

Рис. 4. Параметры $N$ и поляризации при разном числе пластинок толщиной $0.6 \mathrm{~mm}$. $a-N=Q(11) / Q(00) ; b-$ поляризации $P$ и $P_{Q}$
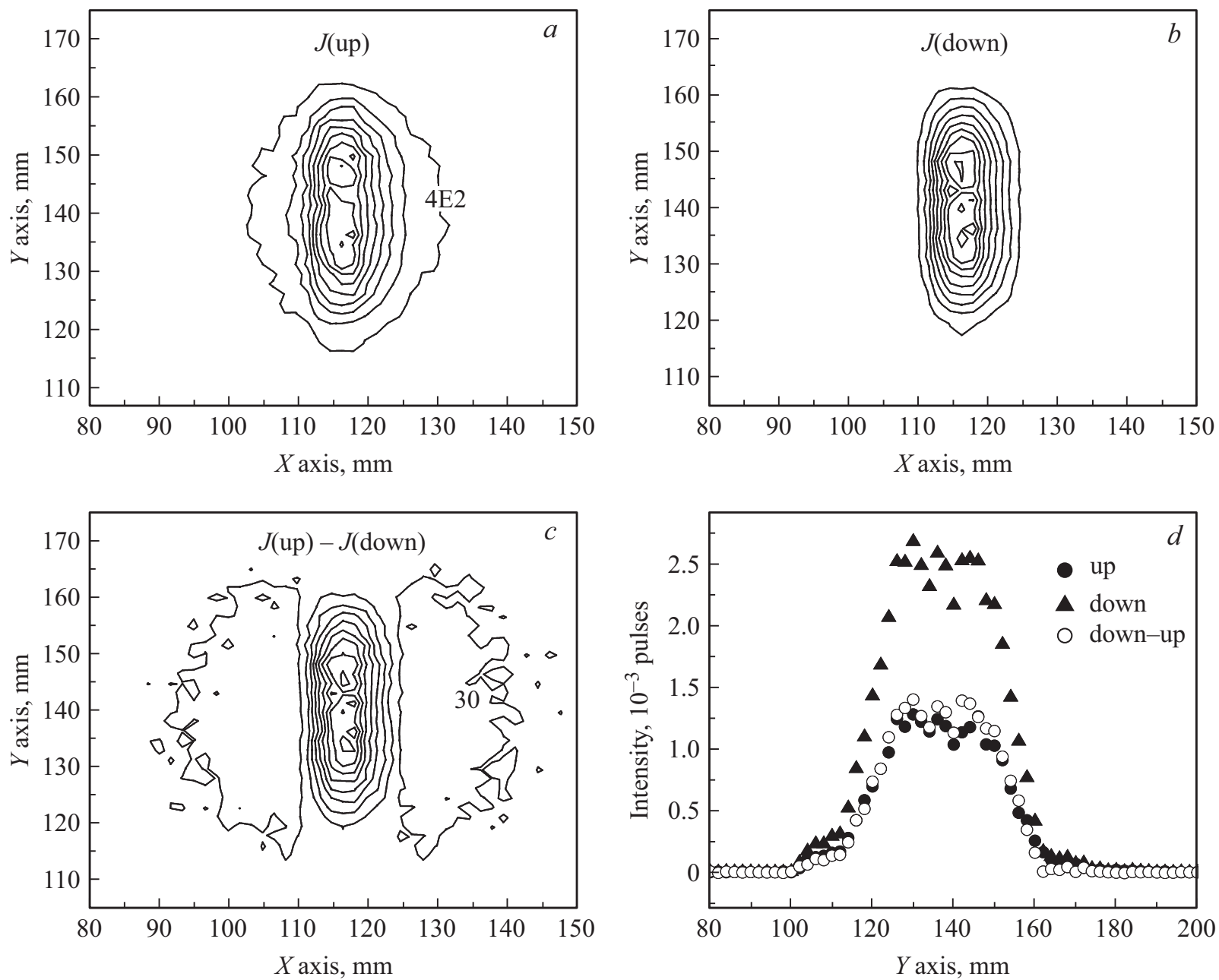

Рис. 5. Интенсивности регистрируемых нейтронов для двух пластинок образца № 1. $(a-c)$ в плоскости $X Y$. $d)$ Вдоль вертикальной оси $Y$ при $X=117 \mathrm{~mm}$.

из четырех пластинок выполнялись измерения при горизонтальном и вертикальном направлениях магнитного поля. О причине полученного при $H=17.6 \mathrm{kOe}$ отли- чия параметров, $N\left(H_{\text {hor }}\right)=1.20(1)$ и $N\left(H_{\text {ver }}\right)=1.14(1)$, будет говориться далее. Как и следовало ожидать, поляризация не зависит от направления $\mathbf{H}$. 

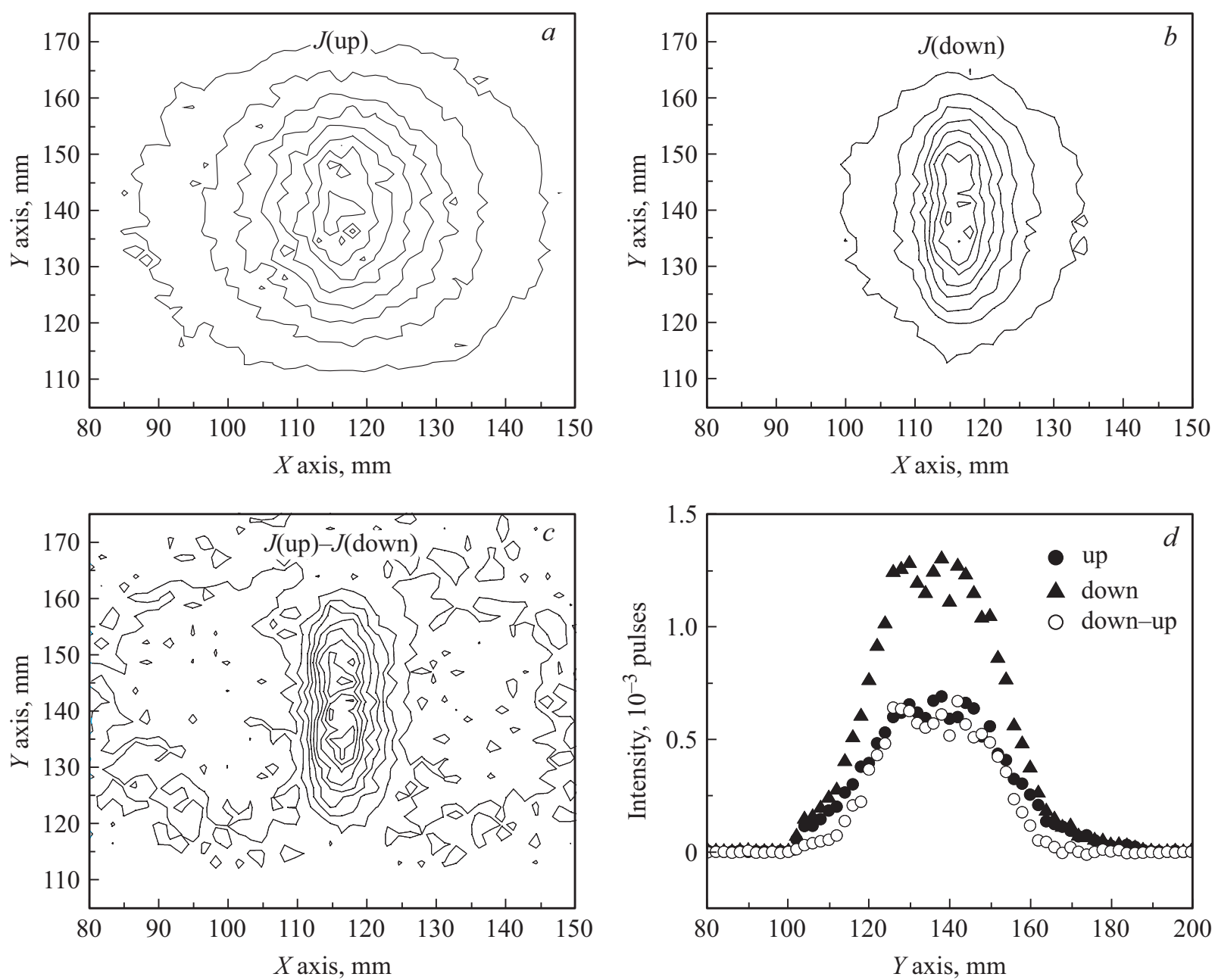

Рис. 6. Интенсивности для образца № 2: $a, b, c-$ в плоскости XY; $d$ - вдоль вертикальной оси $Y$ при $X=117 \mathrm{~mm}$.

3.2. Измерения с использованием двухкоординатного детектора. Образцы намагничивались вертикальным полем $H=13 \mathrm{kOe}$. На рис. 5 показаны линии равных интенсивностей для двух пластинок образца № 1, плоскости которых перпендикулярны направлению нейтронного пучка. Шаг усреднения интенсивностей по направлениям $X$ (горизонталь) и $Y$ составлял $2 \times 2 \mathrm{~mm}$. Для каждого угла $2 \theta$ выполнялось суммирование по координате $Y$ в интервале $104-170 \mathrm{~mm}$ (рис. 5, $d$ ). В таком случае $N=1.207(5)$. Для интервала $\Delta Y=132-144 \mathrm{~mm}$ получилось $N=1.34(1)$. Эти результаты позволяют понять причину отличия величин $N$, полученных ранее при изменении направления Н: при горизонтальном магнитном поле часть МР не регистрируется многоканальной детекторной системой.

Более сильная анизотропия магнитного рассеяния в плоскости $X Y$ наблюдалась для образца № 2 (рис. 6), который $7 \mathrm{~h}$ отжигался при $600^{\circ} \mathrm{C}$ и $14 \mathrm{~h}$ охлаждался в муфельной печи до $170^{\circ} \mathrm{C}$. Для двух пластинок такого образца $N=0.03(1)$, а для интервала $\Delta Y=132-144 \mathrm{~mm}$ $N=0.09(1)$. Измерения на двух образцах, вырезанных из одной заготовки, выполнялись при одних условиях, что позволяет сопоставлять полученные результаты.
Причиной отличия интегральных интенсивностей $Q($ up) и $Q($ down) считалась $[3,4]$ интерференция нейтронных волн в области расходимости первичного нейтронного пучка. Кажется очевидным, что приведенные на рис. 7 результаты для 1-го и 2-го образцов соответствуют такому предположению. Для одной и двух пластинок, вырезанных из одной мишени для магнетронного распыления, получились следующие величины параметра $N$ : для 1-го образца $1.136(5)$ и 1.207 ; для 2-го 1.03(1) и 1.03(1). Для неотожженного образца $W($ up $) \sim W($ down $) \sim W(N r)$, а $N$ равно $1.022(5)$ и $1.042(5)$. Действительные величины $N=Q(11) / Q(00)$, для вычисления которых необходимо знать интегральные интенсивности пиков „с переворотом спина нейтрона“, больше приведенных значений. Понятно, что связанные с этим погрешности величин $N=Q($ down $) / Q($ up $)$ падают с ростом $H$ и растут с толщиной образца.

При определении размеров рассеивающих „частиц“ часто используется разности $[J(\mathrm{up}, 2 \theta)-J($ down, $2 \theta)]$. Однако при анализе кривых $J($ up, $2 \theta)$ и $J($ down, $2 \theta)$ можно получить дополнительную информацию. Сильные отличия кривых рассеяния $J(\mathrm{up}, 2 \theta)$ для двух образцов видны на рис. 8. Но отношения интенсивностей 

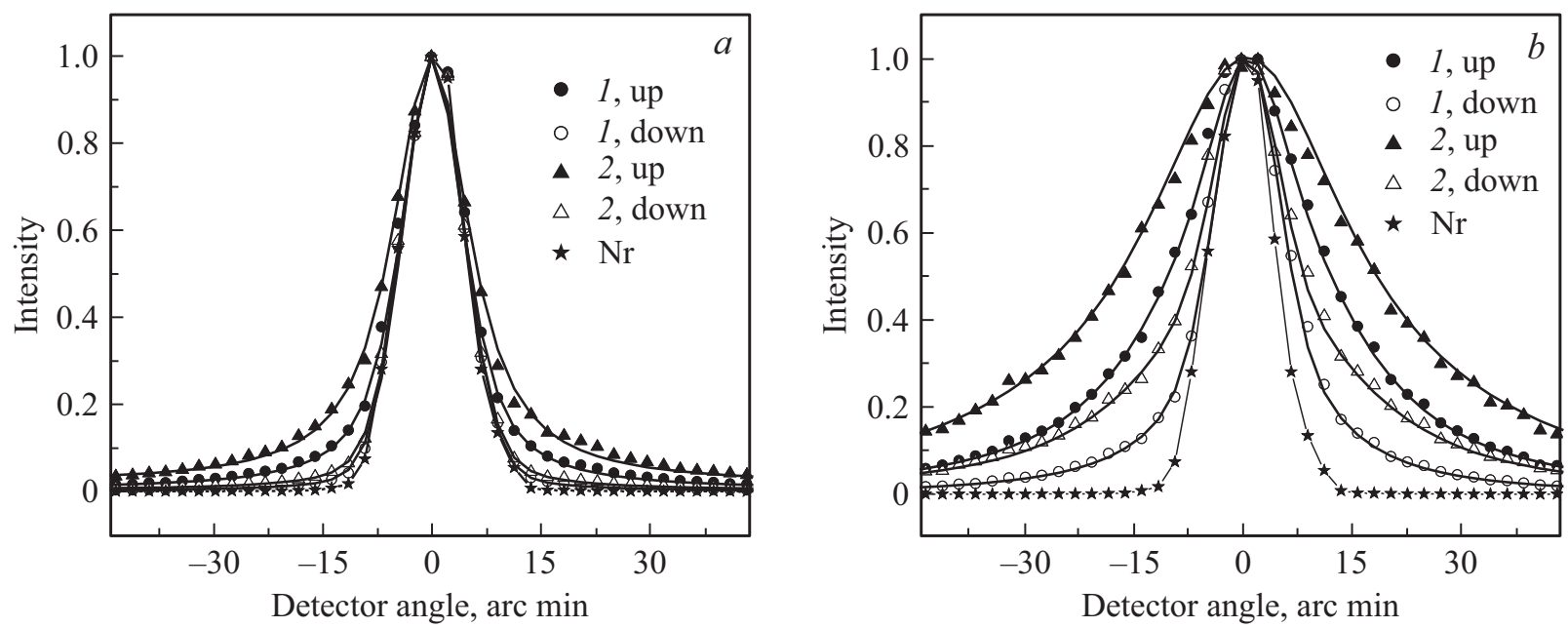

Рис. 7. Угловые распределения интенсивностей вдоль направления $X$ для одной и двух пластинок. $a-$ образец № 1 . $b-$ образец № 2 .
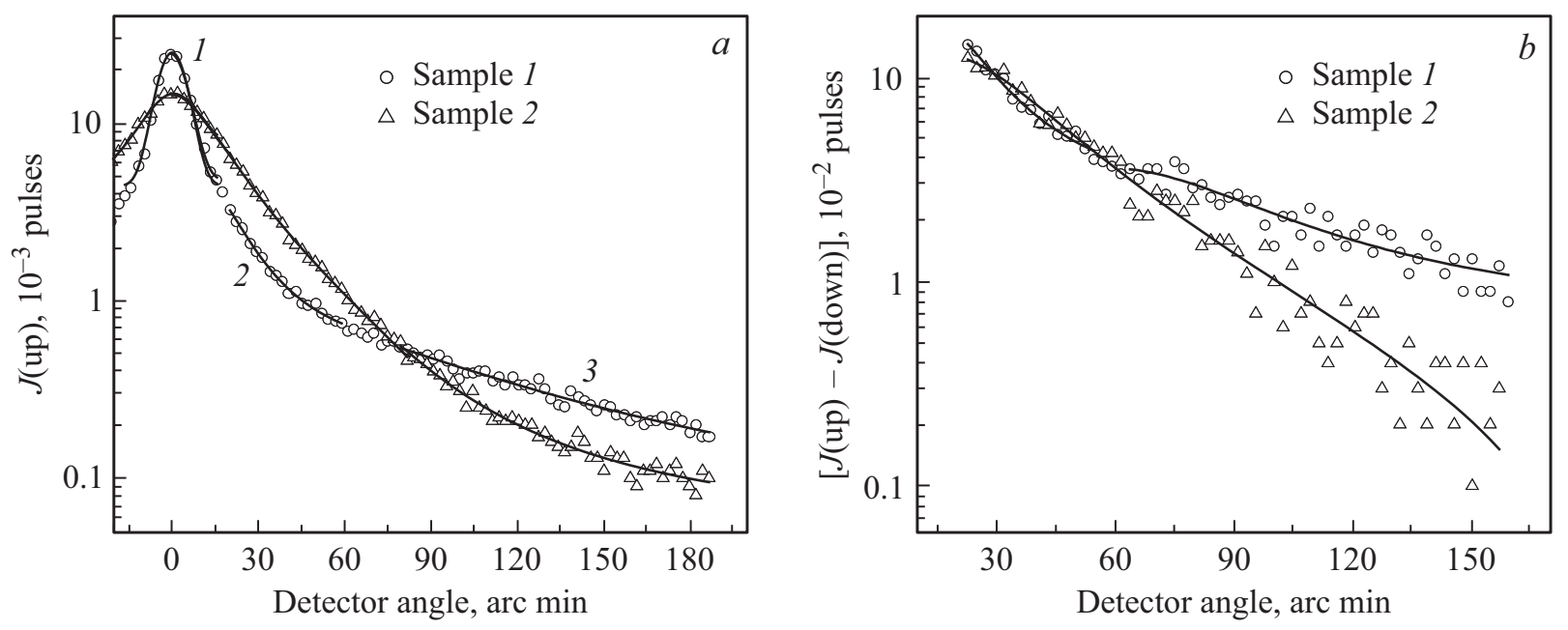

Рис. 8. Угловые распределения интенсивностей для двух пластинок первого $(a)$ и второго $(b)$ образцов.

$Q($ down, 2$) / Q($ down, 1$)=1.02$ и $Q($ up, 2$) / Q($ up, 1$)=1.19$ соответствуют полученным значениям параметров $N$.

Для 1-го образца в области расходимости прямого пучка MP нет (рис. 8, a), так как $W($ up $) \sim W(N r) \sim 11^{\prime}$. Второй и третий интервалы кривой $J(u p, 2 \vartheta)$ аппроксимируются функциями Лоренца с $W=28^{\prime}(2)$ и $W=148^{\prime}(10)$. Для 2-го образца МР наблюдается во всем интервале углов 20: при $2 \theta<20^{\prime} W=29^{\prime}(2)$, а при $2 \theta>20^{\prime} W=40^{\prime}(1)$. Таким образом, величина интерференционного эффекта связана, в основном, с МР на большие углы, что соответствует рис. $8, b$ и рис. $3, b$.

\section{4. Заключение}

Интерференция падающих и рассеянных нейтронных волн при малоугловом рассеянии нейтронов - частный случай квантовых явлений, которые происходят при упругом рассеянии излучения и частиц. Подобные эффекты наблюдаются в разных методах дифракционных и рефлектометрических измерений, результаты которых кажутся простыми и понятными.

Наглядный пример опытов с отражением света плоскопараллельной стеклянной пластинкой приводится в книге [11]. При изменении толщины пластинки сумма интенсивностей отраженного и прошедшего через пластинку света сохраняется, что соответствует самому простому варианту трактовки оптической теоремы. Квантовая механика позволяет легко объяснить периодические изменения интенсивностей, измеряемых детекторами, но механизм „сортировки“ фотонов понять не удается.

В статье [4] имелась ввиду математическая формулировка оптической теоремы [12], физический смысл которой в том, что „интерференция падающей волны с волной, рассеянной на нулевой угол, приводит к выбыванию частиц из падающей волны, что обеспечивает сохранение вероятности“ [13]. При МР также 
должна сохраняться полное сечение упругого рассеяния. Двухкоординатный детектор позволяет регистрировать все нейтроны, рассеянные „вперед“. Разные величины амплитуд рассеяния для двух направлений спина нейтрона приводят к отличию соответствующих потоков нейтронов. Поэтому можно предположить, что результатом интерференции является обратное отношение интенсивностей нейтронов, рассеянных „назад“.

В настоящей работе использовались разные возможности двух типов детекторных систем. Двухкоординатный детектор позволяет получить общую картину МР и выбрать оптимальную схему обработки экспериментальных данных, но измерение поляризации прошедшего через образец пучка необходимо для изучения магнитных текстур и процессов намагничивания.

Автор выражает глубокую благодарность В.В. Рунову за участие в измерениях и М.К. Руновой за возможность использования разработанных ею компьютерных программ.

\section{Список литературы}

[1] A. Wiedenmann. J. Appl. Cryst. 33, 428 (2000).

[2] Sung Ho Lee, Dong Heon Lee, Hyun Jung, Young-Soo Han, Tae-Hwan Kim, Woochul Yang. Carr. Appl. Phys. 15, 915 (2015).

[3] А.В. Ковалев, О.П. Смирнов. ФТТ 55, 81 (2013).

[4] B.P. Toperverg. Physica B 335, 174 (2003).

[5] A.I. Okorokov, V.V. Runov. Physica B 297, 239 (2001).

[6] С.В. Малеев. УФН 172, 617 (2002).

[7] В.В. Рунов, В.С. Ильин, М.К. Рунова, А.К. Раджабов. Письма ЖЭТФ 95, 530 (2012).

[8] А.В. Ковалев. Междунар. научн. конф. „Актуальные проблемы физики твердого тела“. Сб. докл. Минск (2016). T. 1. C. 30.

[9] А.В. Ковалев. ФТТ 53, 669 (2011).

[10] А.В. Ковалев. ФТТ 52, 883 (2010).

[11] Richard P. Feynman. QED The strange Theory of Light and Matter. Princeton University Press, 1985.

[12] Л.Д. Ландау, Е.М. Лифшиц. Квантовая механика. ГИФМЛ, М. (1963). С. 551.

[13] П.В. Елютин, В.Д. Кривченков. Квантовая механика. Наука, М. (1976). С. 169.

Редактор Т.Н. Василевская 